\title{
ELECTRON MICROSCOPY AND X-RAY STRUCTURAL INVESTIGATIONS OF INCOMMENSURATE SPIN-LADDER $\mathrm{Sr}_{4.1} \mathrm{Ca}_{4.7} \mathrm{Bi}_{0.3} \mathrm{Cu}_{17} \mathrm{O}_{29}$ SINGLE CRYSTALS
}

P. Deużewski ${ }^{a}$, A. Pietraszko ${ }^{b}$, M. Kozeowski $^{a}$, A. Szczepańska ${ }^{a}$, J. GóreCKA ${ }^{a}$, M. BARAN ${ }^{a}$, L. LeONYUK $^{c}$, G.-J. BABONAS ${ }^{d}$, O. LeBedev ${ }^{e}$ AND R. SZYMCZAK ${ }^{a}$

${ }^{a}$ Institute of Physics, Polish Academy of Sciences Al. Lotników 32/46, 02-668 Warsaw, Poland

${ }^{b}$ Institute of Low Temperature and Structure Research, 50-422 Wrocław, Poland ${ }^{c}$ Geological Department, Moscow State University, 119899 Moscow, Russia ${ }^{d}$ Semiconductor Physics Institute, 2600 Vilnius, Lithuania

${ }^{e}$ University of Antwerp RUCA, 2020 Antwerp, Belgium

(Received September 4, 2000; in final form October 20, 2000)

Transmission electron microscopy and X-ray diffraction proved chain/ ladder incommensurate single crystal structure of investigated samples. The incommensurate ratio was determined from the X-ray and electron diffraction being equal to 0.704 . Diffuse scattering intensities localised on the planes perpendicular to the $c^{*}$-axis and passing through the spots originating from the periodicity of chain sublattice were detected. High-angle grain boundary or twinning formed by rotation of $33.3^{\circ}$ around [100] direction was observed. High-resolution electron microscopy images revealed the stacking faults in ac planes.

PACS numbers: 74.72.-h, 61.16.Bg, 61.10.-i, 61.72.Nn, 61.72.Mm

\section{Introduction}

The high-temperature superconductivity at $80 \mathrm{~K}$ and at ambient pressure was reported several years ago in the spin-ladder crystals [1] and was confirmed in further studies [2-4]. These layered incommensurate crystals can be described by the formula $\left(\mathrm{A}_{2} \mathrm{Cu}_{2} \mathrm{O}_{3}\right)_{m}\left(\mathrm{CuO}_{2}\right)_{n}$, where $\mathrm{A}$ represents $\mathrm{Sr}$ and $\mathrm{Ca}$ mainly, a small amount of $\mathrm{Bi}$ and sometimes other impurities like $\mathrm{Al}, \mathrm{Pb}$, and $\mathrm{Y}$. The crystal structure of these compounds [5-8] is composed of three kinds of layers: ladders $\left(\mathrm{Cu}_{2} \mathrm{O}_{3}\right)$, $\mathrm{Sr} / \mathrm{Ca}$ cation layer, chains $\left(\mathrm{CuO}_{2}\right)$. The structure of $\left(\mathrm{A}_{2} \mathrm{Cu}_{2} \mathrm{O}_{3}\right)_{m}\left(\mathrm{CuO}_{2}\right)_{n}$ system can be interpreted as two interpenetrating orthorhombic sublattices 
$\left(\mathrm{A}_{2} \mathrm{Cu}_{2} \mathrm{O}_{3}\right)$ and $\left(\mathrm{CuO}_{2}\right)$ with common lattice parameters along the $a$ and $b$ axes. In these sublattices the parameters along the $c$ axis, $c_{L}$ and $c_{C}$, are incommensurate. The common lattice parameter $c$ for the best commensurate approximation can be written as $c=m c_{L}=n c_{C}$, where $m$ and $n$ are integers and the $m / n$ ratios can be equal to $5 / 7$ and $7 / 10$, respectively. This corresponds to chemical formulas $\mathrm{A}_{10} \mathrm{Cu}_{17} \mathrm{O}_{29}$ and $\mathrm{A}_{14} \mathrm{Cu}_{24} \mathrm{O}_{41}$, respectively.

It should be mentioned that simultaneously with the first report [1] the superconductivity below $12 \mathrm{~K}$ was also reported in $\mathrm{Bi}$-free $(\mathrm{Sr}, \mathrm{Ca})_{14} \mathrm{Cu}_{24} \mathrm{O}_{41}$ spin-ladder crystals under hydrostatic pressure of $3-4.5 \mathrm{GPa}$ [9]. The presence of a small amount (less than 1 at.\%) of bismuth in the investigated in the present work single crystals has born a suspicion that superconductivity observed in these samples could be connected with precipitation of the $\mathrm{Bi}_{2} \mathrm{Sr}_{2} \mathrm{CaCu}_{2} \mathrm{O}_{8+\delta}$ phase. It is well known that the $\mathrm{Bi}_{2} \mathrm{Sr}_{2} \mathrm{CaCu}_{2} \mathrm{O}_{8+\delta}$ phase has also $T_{\mathrm{c}}$ close to $80 \mathrm{~K}$ and consists of the same elements. Nevertheless, the magnetization measurements have shown that at least $10 \%$ of crystal volume exhibits the transition to superconducting state at the temperature of about $80 \mathrm{~K} \mathrm{[10].} \mathrm{It} \mathrm{is} \mathrm{distinctly} \mathrm{larger} \mathrm{than} \mathrm{a} \mathrm{possible}$ volume of $\mathrm{Bi}_{2} \mathrm{Sr}_{2} \mathrm{CaCu}_{2} \mathrm{O}_{8+\delta}$ phase, which follows from the chemical composition.

On the other hand, the presence of $\mathrm{Bi}$ atoms could be a reason of unique structural properties of these crystals. It is possible that $\mathrm{Bi}$ atoms induce local structural defects in nano- and atomic scale responsible for the electronic properties different from those for Bi-free crystals. The transmission electron microscopy and diffraction seems to be a proper tool for examination of the crystal structure in nano- and atomic scale.

\section{Experimental}

The investigated crystals were grown by the "melted band" method [11] using $\mathrm{Bi}_{2} \mathrm{CuO}_{4}$ as a flux. The crystals possessed a needle-like shape along the $c$-axis. They are black coloured with shiny metallic surfaces.

$\mathrm{X}$-ray measurements were carried out using KUMA DIFFRACTION single crystal diffractometer equipped with a two-dimensional area CCD detector. The Mo $K_{\alpha}$ graphite-monochromated radiation and the omega scan with $D w=0.75^{\circ}$ and the time of exposition $t=45 \mathrm{~s}$ for single image was used for data collection. The 1400 images for 9 different runs (with two $\theta$ positions of CCD detector, $\theta=31^{\circ}$ and $\theta=70^{\circ}$ ) covered $95 \%$ of the Ewald sphere. The lattice parameters were calculated using about 250 reflections obtained from 30 images for 10 runs with different orientations in reciprocal space. The structure was solved by a heavy-atom method and subsequent difference Fourier syntheses with a help of SHELXTL_PLUS program system [12]. The structure refinement by a full matrix, least squares method on $F_{0}^{2}$ was done using SHELX97 [13].

The specimens for transmission electron microscopy (TEM) were prepared by ion milling and crushing methods. Using the crushing method, the sample was powdered in an agate mortar, dispersed ultrasonically in butyl alcohol and deposited onto holey carbon film supported on $\mathrm{Cu} 300$ mesh grid. In the case of ion milling, the needle-like crystals were mounted to $3 \mathrm{~mm}$ brass discs in two variants. In the first variant, the needle axis was parallel to the disc surface and in 
the second one it was perpendicular to the disc. Next, the discs were mechanically polished and dimpled to a thickness less than 40 microns and finally both sides were milled by $\mathrm{Ar}$ ion beams with 4-6 keV energy and $12^{\circ}$ inclined to the discs surface.

TEM investigations were made using JEM 2000EX as well as JEM-4000 microscope operating at $200 \mathrm{kV}$ and $400 \mathrm{kV}$, respectively. The diffraction patterns and high resolution TEM (HRTEM) images were interpreted with the aid of EMS P. Stadelman software package [14]. JEOL JXA-50A microprobe with WDA detector and electron beam with $20 \mathrm{keV}$ energy and 1 micron probe size was applied for determination of chemical composition homogeneity.

\section{Results}

Using optical and electron probe microanalysis it was found that the samples under investigation were homogeneous in the scale of microns. For specimen's surface perpendicular to the needle axis a set of parallel sharp lines was observed under optical microscope. Electron-probe microanalysis (EPMA) did not show any differences in the intensities of $\mathrm{X}$-ray characteristic radiation of $\mathrm{Sr}, \mathrm{Cu}, \mathrm{Ca}$, and $\mathrm{Bi}$ in the line region and outside of it. From these measurements the relative atomic concentrations of all cations $\mathrm{Cu}, \mathrm{Ca}, \mathrm{Sr}, \mathrm{Bi}$ were determined as 65.0:17.9:15.8:1.3, respectively.

X-ray single crystal diffraction measurements reveal a high quality of investigated crystals. The reconstruction of reflection positions in reciprocal space shows two distinctly different orthorhombic sublattices with the common $a^{*}$ and

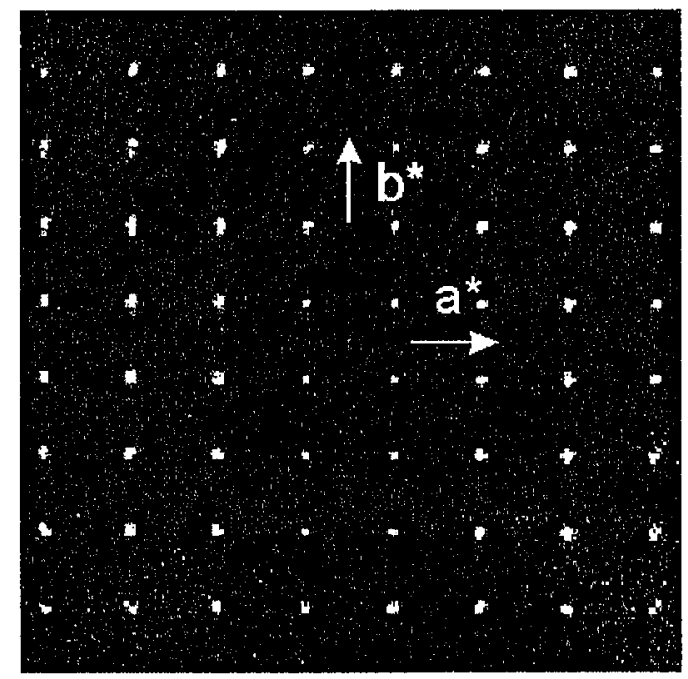

Fig. 1. The reconstructed $h k 7$ zone showing a diffuse scattering. The intensities along the $b^{*}$-axis are stronger than those along the $a^{*}$-axis. 

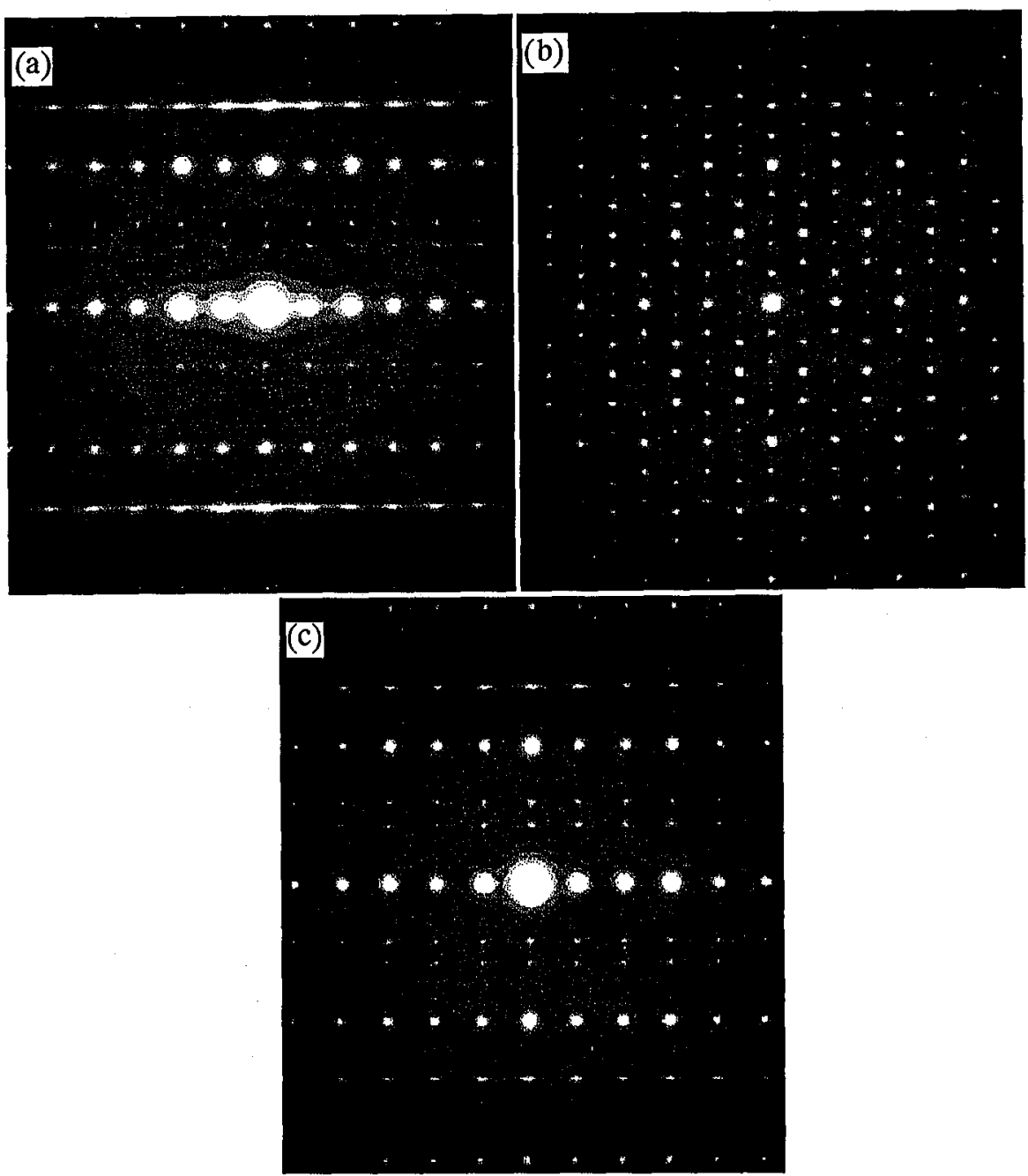

Fig. 2. Electron diffraction patterns along (a) [100], (b) [110] and (c) [010] zone.

$b^{*}$ but with different $c^{*}$ parameters. The calculated lattice constants in the real space are as follows: $a=11.38971 \pm 0.00159 \AA, b=12.96153 \pm 0.00169 \AA$, $c_{L}=3.91793 \pm 0.00061 \AA, c_{C}=2.75931 \pm 0.00063 \AA$. The two commensurate approximations with $m / n=5 / 7$ and $7 / 10$ were used for solving the crystal structure. The $R$-factor was equal to $5.5 \%$ and $7.5 \%$, respectively. The diffuse scattering along the $a^{*}$ and $b^{*}$ directions was detected for the reflections originating from the chain sublattice (Fig. 1).

The transmission electron diffraction and microscopic investigations were performed for samples prepared in two configurations: (i) specimens with needle axis parallel to the surface, and (ii) specimens with needle axis perpendicular to the surface. For the first case the typical electron diffraction patterns are presented 
in Fig. 2. The patterns were indexed according to the orthorhombic $F 222(\mathrm{~m} / \mathrm{n}=$ $5 / 7)$ structure [15]. No difference in electron diffraction patterns was observed for the samples prepared by the crushing and ion milling methods in contrast to results presented in [16]. The ratio of the $c$ parameters for the ladder and chain sublattices was determined from the length of $\left(\begin{array}{lll}0 & 0 & 10\end{array}\right)$ and $\left(\begin{array}{lll}0 & 0 & 14\end{array}\right)$ diffraction vectors on [100], [110], and [010] diffraction zones and was found to be $0.703 \pm 0.001$. Besides the Bragg diffracted spots, intensities of diffuse scattering were observed. The intensities were localised on the lines perpendicular to $c^{*}$ direction and passed through the spots originating from the chain sublattice. It was characteristic that diffuse intensities were more pronounced on [100] and [010] zones than on [110] diffraction zone.

Electron microscopy of the specimens with needle axis perpendicular to the surface showed the regions of perfect crystal structure. In several cases the regions with characteristic linear defects were observed. The defects had the form of stripes spread along the [100] direction. The stripes had various widths from several (020) interplanar spacings to hundreds of nanometers. Figure 3 presents the region with relatively large amount of defects with the widths of several (020) spacing. The diffraction pattern obtained from this region exhibited the lines of diffuse intensities along the $b^{*}$ direction passing through $(020)$ reflections. The defects introduced a half distance shift of (200) or/and (020) interplanar distance, or did not introduce the shift at all. It was found that defects introduced the shift in the (020) planes exclusively or in the (200) and (020) planes simultaneously. The situation with the shift occurring only in the (200) planes was not observed at all.

Figure 4 presents the diffraction image observed in the region of defects exceeding several nanometers in size. The set of diffraction spots was a superposition
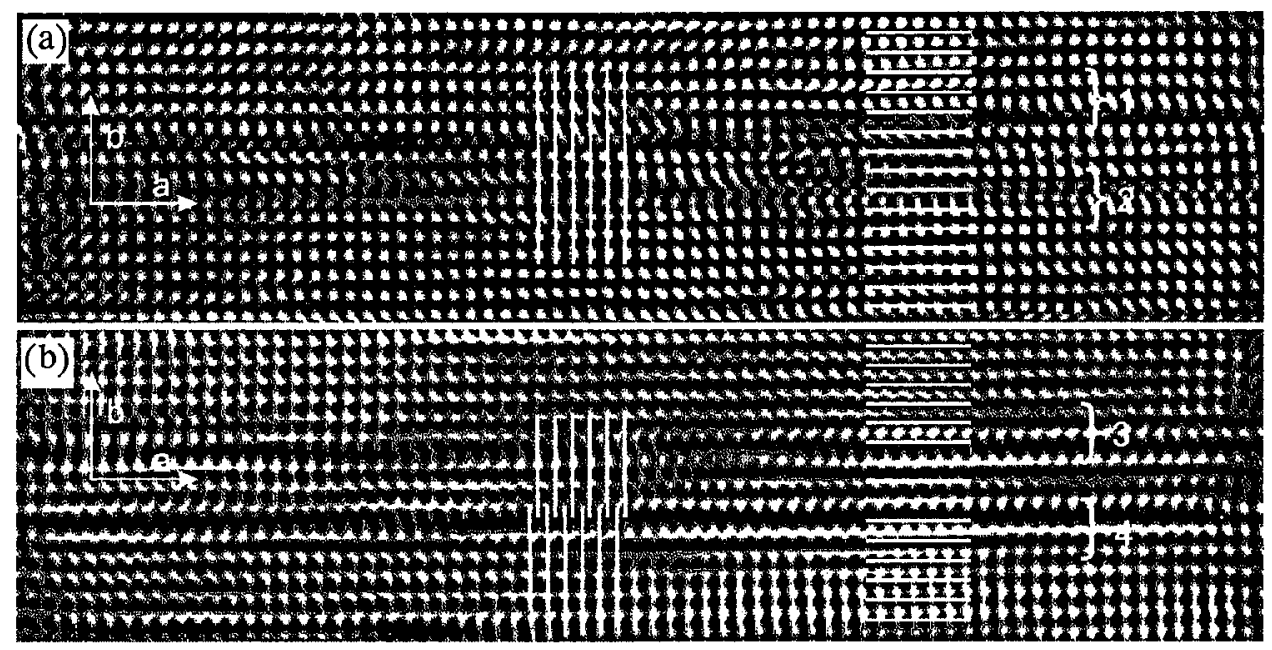

Fig. 3. High-resolution [001] zone images, (a) and (b), exhibiting various stacking faults (1-4). The disturbances in the arrangement of the (020) planes $(1,3)$ and in both (020) and (200) planes (4) are shown. The arrangement of the planes is conserved in (2). 

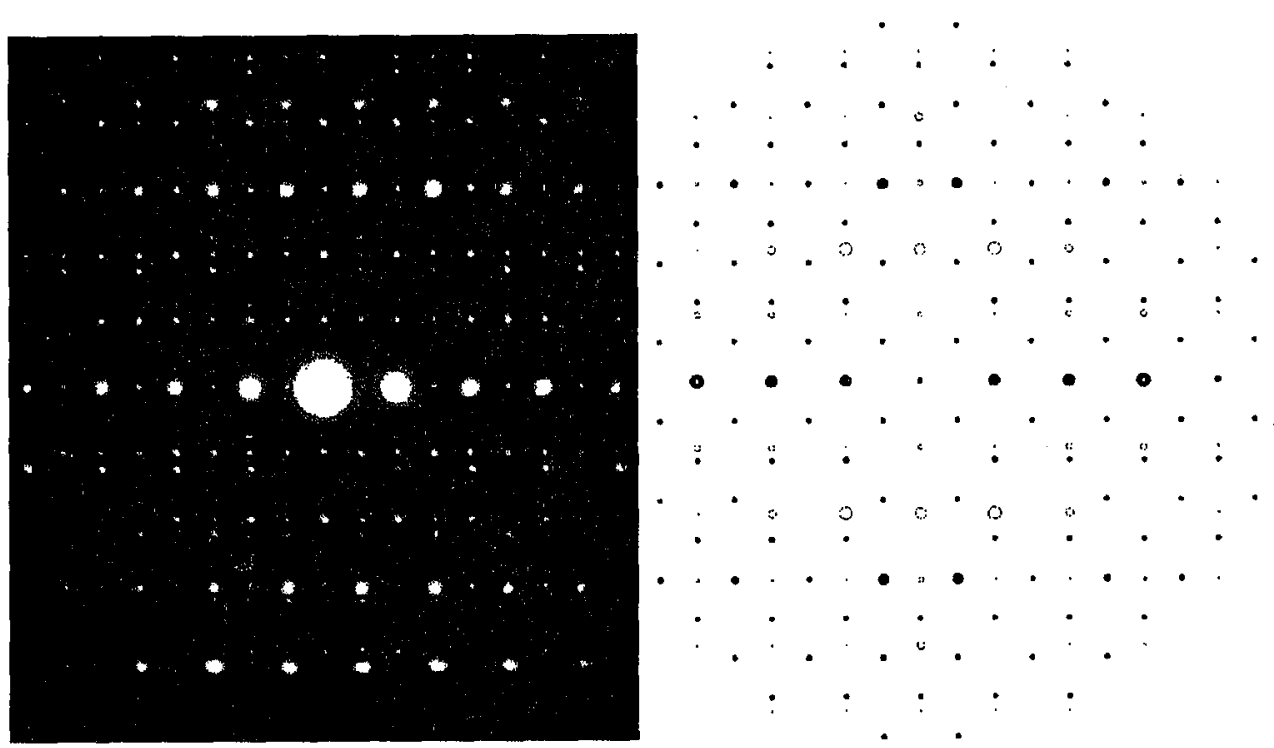

Fig. 4. The typical double diffraction of [001] (open circles) and [011] (solid circles) zones.

of [001] and [011] diffraction patterns belonging to the same crystal structure. Both diffraction patterns have the same $a^{*}$ axis and can be transformed one to another by the $33.3^{\circ}$ rotation around the $a^{*}$-axis. Dark-field observations proved that these diffraction patterns originated from different crystal blocks.

\section{Discussion}

The X-ray and electron diffraction confirmed the chain/ladder incommensurate crystal structure of the samples under investigations. The $m / n=0.70428$ calculated as the $c_{C} / c_{L}$ ratio was between those for commensurate approximations $5 / 7=0.714$ and $7 / 10=0.7$. Although the obtained $m / n$ value is closer to that expected for $7 / 10$, nevertheless the obtained values of refinement factor $(R)$ indicate rather on $5 / 7$ as a better approximation of the investigated structure. Taking this into account and using the EPMA results, the chemical formula of the investigated crystals can be written as $\mathrm{A}_{9.2} \mathrm{Cu}_{17} \mathrm{O}_{29}$ (where $\mathrm{A}_{9.2}=\mathrm{Sr}_{4.1} \mathrm{Ca}_{4.7} \mathrm{Bi}_{0.3}$ ). Some deficit in the $A$ positions should be noticed in comparison to the $\mathrm{A}_{10} \mathrm{Cu}_{17} \mathrm{O}_{29}$ formula following from the $5 / 7$ commensurate approximation.

The observed diffused scattering and the mentioned above deficit in the A positions strongly suggest the existence of structural defects. For the interpretation of diffuse scattering, the "initial phase disorder" model of the chain/ladder structure by Wu et al. [16] and the "pencil disorder" structural model by Milat et al. [17] were developed. In both models, there is assumed that $\mathrm{Cu}-\mathrm{O}$ chains are weakly spatially correlated. Recently, Ammerahl and Revcolevschi [18] proposed the one-dimensional initial phase disorder along the $a$-direction for interpretation of diffraction streaks observed in X-ray studies on spin-ladder compounds 
$(\mathrm{Ca}, \mathrm{La})_{14} \mathrm{Cu}_{24} \mathrm{O}_{41}$. The latter model assumes a different degree correlation of chains for various directions. It seems that this model could be used to explain also the observed strongest diffused scattering along the $b^{*}$-axis (Fig. 2).

The linear defects observed on the [001]-oriented samples suggest a formation of the stacking faults in the stacking arrangement of ladder and chain planes. One possible interpretation of these defect structures is based on the assumption that the defects form additional chain or ladder planes. In perfect spin-ladder crystal structure the stacking sequence of the $a c$ planes is as follows ...CALAcalaCALA..., where $C$ and $c$ indicate chain planes, $A$ and $a-$ cation planes, $L$ and $l$-ladder planes. Any fault of the staking sequence would be seen as a disturbance of (020) lattice fringes observed on high-resolution electron images. One (020) fringe corresponds to the layer composed of the cala or $C A L A$ planes. In the situation, when $L A$ (or $l a$ ) planes are removed, the stacking sequence is ...CAcalaCALA... (or ...CALAcaCALA ...).

The removal of $l a$ or $L A$ planes results in a shift of lattice fringes by $\frac{1}{4} b$. Similar situation takes place, when $L A$ (or $l a$ ) planes are added. This was observed on high-resolution images taken along [001] zone. In Fig. 3a one can see that (200) lattice fringes remain unchanged on both sides of the stacking faults. When even number of $L A$ (or $l a$ ) planes are added, the (020) fringes should be shifted by even number of $\frac{1}{4} b$. Therefore, the shift could not be observed on HRTEM image along the $c$ direction. The joint shift of the (020) and (200) lattice fringes was also observed (Fig. $3 \mathrm{~b}$ ). In this case a simple addition or removal of set of planes is not sufficient to explain the extra shift in the $a$ direction and a more complex presumption is necessary. It is conceivable that additional $L A$ (or la)

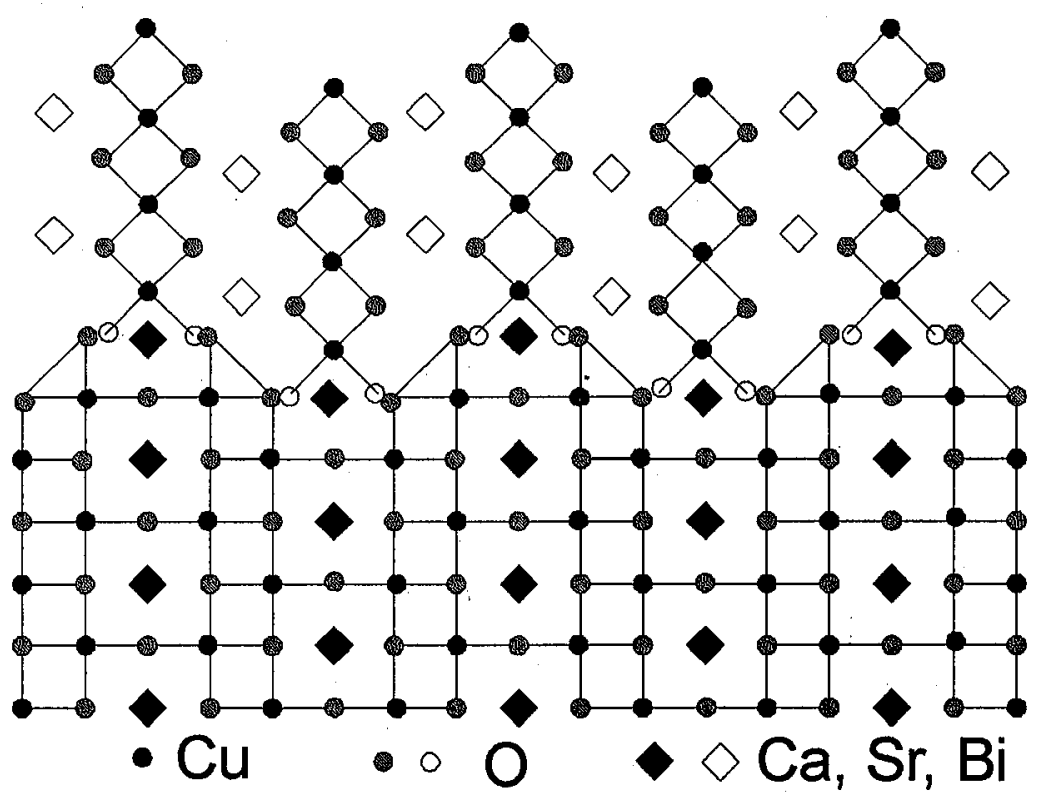

Fig. 5. The proposed model of transformation of the ladder plane to the chain plane. 


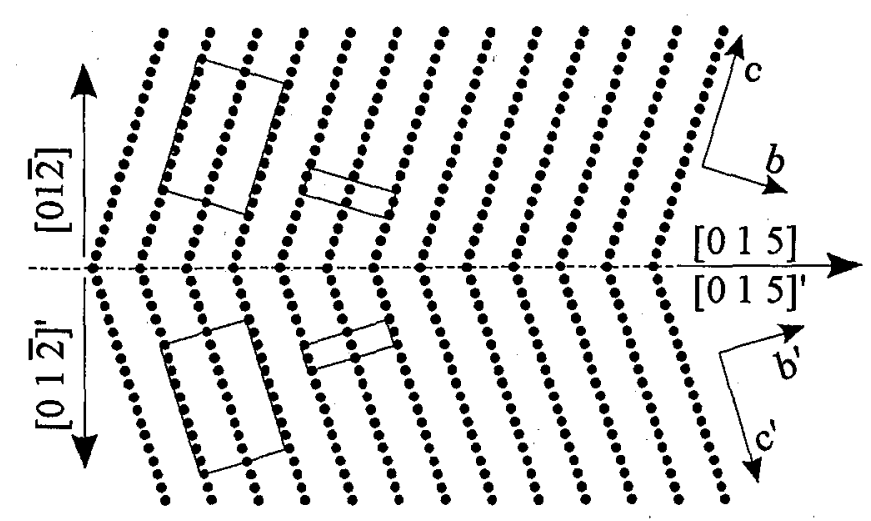

Fig. 6. Schematic drawing of the twinning system, which fulfils the crystallographic relationship deduced from the diffraction pattern presented in Fig. 4. For simplicity, only the ladder planes are shown.

planes transform themselves to the chain plane or vice versa. In Fig. 5 a possible model of transformation from the ladder to chain plane is shown. The proposed transformation explains the shifts of both (200) and (020) lattice fringes.

The stripes with a width of several and more nanometers consist of blocks with identical chain/ladder structure. The blocks can be transformed one to another by $33.3^{\circ}$ rotation around the common $a$-axis. Such a crystallographic relationship suggests a twining system with $\left(\begin{array}{lll}0 & 1 & 2\end{array}\right)$ (Fig. 6) or $\left(\begin{array}{lll}0 & 5 & 1\end{array}\right)$ mirror plane.

A local stress and/or a change of chemical composition could introduce a transformation leading to the formation of linear defects observed on TEM images as the thin and wide stripes. It is possible to assume that these defects are the result of a relatively high density of the point defects situated in the investigated crystals. However, the nature of these defects is difficult for resolving at the present stage of investigations.

The absence of manifestation of these defects for the specimen with the surface parallel to the $c$-axis is still an open question.

\section{Conclusions}

The $\mathrm{X}$-ray and TEM investigations proved the incommensurate chain/ladder structure of the investigated crystals. Both methods revealed the high quality of these crystals. The structure refinement indicated the $\left(\mathrm{A}_{2} \mathrm{Cu}_{2} \mathrm{O}_{3}\right)_{5}\left(\mathrm{CuO}_{2}\right)_{7}$ system as the best commensurate approximation for the investigated material. The diffraction patterns have shown diffuse scattering originating from a relatively high disorder in the chain sublattice. The TEM observation revealed high-angle grain boundaries or twining formed by rotation of $33.3^{\circ}$ around the $a$ direction. The stacking faults of two different types were found in high-resolution images. The grain boundary and stacking faults could be caused by a condensation of point defects situated in the investigated crystals. 


\section{Acknowledgments}

The work was partly supported by the Committee for Scientific Research (Poland) under grant No. 2 P03B 102 14. One of us (L.L.) acknowledges U.S. Civilian Research and Development Foundation (project CRDF 6422) for financial support in crystal growth experiment. P.D. would like to thank Prof. G. Van Tendeloo for fruitful discussion.

\section{References}

[1] L. Leonyuk, G.-J. Babonas, A. Vasilev, R. Szymczak, A. Reza, V. Maltsev, L. Ponomarenko, Czech J. Phys. 46, Suppl. S3, 1457 (1996).

[2] M. Baran, R. Szymczak, H. Szymczak, L. Leonyuk, G.-J. Babonas, Adv. Sci. Technol. 23, 585 (1999).

[3] M. Baran, R. Szymczak, H. Szymczak, E. Mosiniewicz-Szablewska, L. Leonyuk, G.-J. Babonas, V. Maltsev, J. Magn. Magn. Mater. 197, 527 (1999).

[4] A.I. D'yachenko, V.Yu. Tarenkov, R. Szymczak, A.V. Abal'oshev, I.S. Abal'osheva, S.J. Lewandowski, L. Leonyuk, Phys. Rev. B 61, 1500 (2000).

[5] V.K. Kato, E. Takayama-Muromachi, K. Kosuda, Y. Uchida, Acta Crystallogr. $C$ 44, 1881 (1988).

[6] S.T. Siegrist, L.F. Schneemeyer, S.A. Sunshine, J.V. Waszczak, R.S. Roth, Mater. Res. Bull. 23, 1429 (1988).

[7] E.M. McCarron, M.A. Subramanian, J.C. Calabrese, R.L. Harlow, Mater. Res. Bull. 23, 1355 (1988).

[8] T. Ohta, F. Izumi, M. Onoda, M. Isobe, E. Takayama-Muromachi, A.W. Hewat, J. Phys. Soc. Japan 66, 3107 (1997).

[9] M. Uehara, T. Nagata, J. Akimitsu, H. Takahashi, N. Mori, K. Kinoshita, J. Phys. Soc. Japan 65, 2764 (1996).

[10] R. Szymczak, H. Szymczak, M. Baran, E. Mosiniewicz-Szablewska, L. Leonyuk, G.-J. Babonas, V. Maltsev, L. Shanskaya, Physica C 311, 187 (1999).

[11] V. Maltsev, L. Leonyuk, G.J. Babonas, R. Szymczak, A. Reza, J. Cryst. Growth 211, $501(2000)$.

[12] G.M. Sheldrick, SHELXL, Program for Crystal Structure Refinement, University of Goettingen, Germany 1993.

[13] G.M. Sheldrick, SHELXL, Program for Crystal Structure Refinement, University of Goettingen, Germany 1997.

[14] P.A. Stadelman, Ultramicroscopy 21, 131 (1987).

[15] L. Leonyuk, G.-J. Babonas, V. Rybakov, E. Sokolova, R. Szymczak, V. Maltsev, L. Shvanskaya, J. Phys. Chem. Solids 59, 1591 (1998).

[16] X. Wu, E. Takayama-Muromachi, S. Suehara, S. Horiuchi, Acta Crystallogr. A 47, 727 (1991).

[17] O. Milat, G. Van Tendeloo, S. Amelinckx, M. Mehbod, R. Deltour, Acta Crystallogr. A 48, 618 (1992).

[18] U. Ammerahl, A. Revcolevschi, J. Cryst. Growth 197, 825 (1999). 